\title{
Sequential Administration of EGFR-TKI and Pemetrexed Achieved a Long Duration of Response in Advanced NSCLC Patients with EGFR-mutant Tumours
}

\author{
Chen Yue-Yun, Hong Ye, Fu Yang, Li Qing, Lin Pan-Pan, Ding Zhen-Yu*
}

\begin{abstract}
Objectives: The optimal combination of epidermal growth factor receptor (EGFR) tyrosine kinase inhibitors (TKIs) and chemotherapy has helped to improve therapeutic effects in non-small-cell lung cancer (NSCLC). This study aimed to explore the progression free survival (PFS) of patients after sequential administration of TKI and pemetrexed chemotherapy. Methods: This study retrospectively screened treatment-naive advanced NSCLC patients harbouring EGFR mutations who were prescribed a TKI and salvaged with pemetrexed chemotherapy or vice versa. The total, initial and salvage PFS were collected. Results: The total PFS including both the initial and salvage PFS was 18.0 mon (95\% CI: 14.1-21.9 mon), which was not influenced by the sequence of administration (TKI first: 18.0 mon, 95\% CI: 15.8-20.2 mon, pemetrexed first: $16.1 \mathrm{mon}, 95 \%$ CI: 9.1-23.1 mon, HR 0.92, $\mathrm{P}=0.748)$. A longer PFS was achieved for TKI over chemotherapy in both the initial (10.6 and 5.9 mon, HR 2.62, $\mathrm{P}=0.001)$ and salvage therapy $(12.0$ and 6.0 mon, HR 1.29, $\mathrm{P}=0.001)$. TKI remained effective either before (10.6 mon) or after (12.0 mon) chemotherapy (HR 0.96, $\mathrm{P}=0.853$ ). The same trend was observed for chemotherapy (5.9 and 6.0 mon for initial and salvage therapy, respectively, HR 0.82, $\mathrm{P}=0.417$ ). Conclusions: The sequential administration of TKI and pemetrexed chemotherapy achieved a long PFS and was a suitable treatment for advanced NSCLC.
\end{abstract}

Keywords: NSCLC- EGFR-TKI- sequential administration- pemetrexed- PFS

Asian Pac J Cancer Prev, 20 (8), 2415-2420

\section{Introduction}

\section{Background}

Currently, lung cancer ranks No. 1 in both morbidity and mortality worldwide (Hirsch et al., 2009; Siegel et al., 2018). Non-small-cell lung cancer (NSCLC) constitutes approximately $80 \%$ of lung cancer cases. Most patients have a poor prognosis, and the 5 -year survival is estimated to be only approximately $15 \%$, despite the adoption of aggressive therapies (Torre et al., 2016). Progress in treating NSCLC was achieved recently. In non-squamous cancer, pemetrexed-based doublet chemotherapy achieved superior effects (Wu et al., 2014). In addition, studies have suggested treatments with epithelial growth factor receptor tyrosine kinase inhibitors (EGFR-TKIs) are more efficacious over chemotherapy in selected patients harbouring EGFR mutations (Paez et al., 2004; Zhou et al., 2015; Cheng et al., 2016; Mok et al., 2009). Efforts were devoted to combine these two efficacious therapies together (Lee et al., 2013; Yang et al., 2016). In the pilot JMIT study, the progression-free survival (PFS) of a combination of gefitinib and pemetrexed (15.8 months) was better than that of gefitinib alone (10.9 months).
However, the possible benefits of pemetrexed were excluded in the gefitinib arm in this study, i.e., the effects of the sequential administration of TKI and pemetrexed were not seriously evaluated.

To explore the outcome of their sequential administration, a retrospective study was performed. We screened 1,682 consecutive NSCLC patients treated from 2012 to 2017 and selected 94 patients for the current analysis.

\section{Materials and Methods}

\section{Study Population}

This retrospective study was conducted on patients admitted to the West China Hospital from March 2012 to Jan 2017, and consecutive patients were screened against the inclusion criteria, namely, pathologically confirmed, metastatic (stage IV according to the 7th edition AJCC cancer staging manual), treatment-naive NSCLC patients harbouring sensitive EGFR mutations (exon 19 deletion, exon 21 L858R missense mutation, or others) were who were prescribed an EGFR TKI and salvaged by pemetrexed-based doublet chemotherapy upon tumour 
progression (Progressive Disease, PD) (Rice et al., 2010; Eisenhauer et al., 2009). In addition, patients who received these two treatments in a reverse sequence, i.e., pemetrexed doublet chemotherapy salvaged by EGFR TKI, were also included. Exclusion criteria were patients $\leq 18$ years old, existing secondary malignancy, presence of primary resistance mutation (T790M), or complex small-cell lung cancer. Local palliative radiotherapy for bone or brain metastases was allowed. The ethical committee of Sichuan University reviewed the study concept and the study was performed in accordance with the Declaration of Helsinki.

\section{Treatment Protocol}

Pemetrexed (Lily, IL) was intravenously infused at a dose of $500 \mathrm{mg} / \mathrm{m}^{2}$ on day 1 of each 21-day cycle, supplemented with folic acid, vitamin B12, and dexamethasone, as per the pemetrexed prescribing information. Gefitinib (250 mg, AstraZeneca, UK) and erlotinib (150 mg, Roche, Switzerland) were both taken orally once per day, and icotinib (125 mg, Beta, China) was taken 3 times a day. Treatment continued until disease progression, unacceptable toxicity, or another situation occurred for which it was considered inappropriate to continue.

\section{Outcome Measures}

Tumours were assessed regularly every 2 months radiographically, including computed tomography of the chest and upper abdomen, magnetic resonance imaging of the head, and bone scintigraphy. The tumour response was determined by the treating physicians. Tumour response was described as a complete response (CR), a partial response (PR), stable disease (SD), or progressive disease (PD) based on the RECIST 1.1. The total PFS (PFSt) was defined as the duration from the initiation of the therapy to the date of first onset of PD during the salvage therapy. PFSt included both the PFS of the initial therapy (either chemotherapy, PFS1c, or TKI, PFS1t) and the salvage therapy (chemotherapy, PFS2c, or TKI, PFS2t).

\section{Statistical Analysis}

Statistical analysis was performed using SPSS version 22.0 (IBM Inc., Chicago, IL) and the results of the multi-variate analysis were output by Stata MP 14 (Stata Corp LP., Chicago, IL). The quantitative data were compared using chi-square tests and Fischer's exact test according to Cochran's rule. Kaplan-Meier curves were used to compare survival. Multi-variate analysis was conducted by using a Cox proportional hazard model. All P-values were based on a two-tailed hypothesis, and statistical significance was assumed if $\mathrm{p}<0.05$.

\section{Results}

\section{Patient characteristics}

A cohort of consecutive 1,682 patients admitted during the time period was screened for inclusion. Those with an earlier stage (I-III) without a sensitive EGFR mutation, including unknown mutation status, treated with chemotherapy other than pemetrexed, or failure to accomplish both lines of therapies, were excluded. A total of 94 patients were thus enrolled in this study. Their demographic features are summarized in Table 1 . The majority were women, non-smokers, had adenocarcinoma and were younger $(<60)$.

\section{Total PFS}

The total PFS of the whole cohort, irrespective of administration sequence, was 18.0 mon (95\% CI: 14.1-21.9 mon, Figure $1 \mathrm{~A}$ ). Multivariate analysis indicated that subgroups including age, sex, smoking history, histology, PS score, and EGFR mutation type did not influence the PFS independently (Figure 2).

\section{Therapeutic effects between variant administration sequence}

Patients were grouped according to the sequence of chemotherapy and TKI. Those with initial chemotherapy (CT group) were comparable to those of the reverse sequence of therapy (initial TKI therapy, TC group) in
A

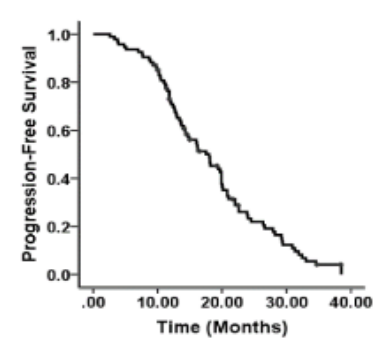

C

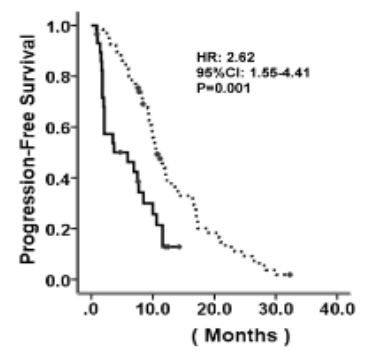

E
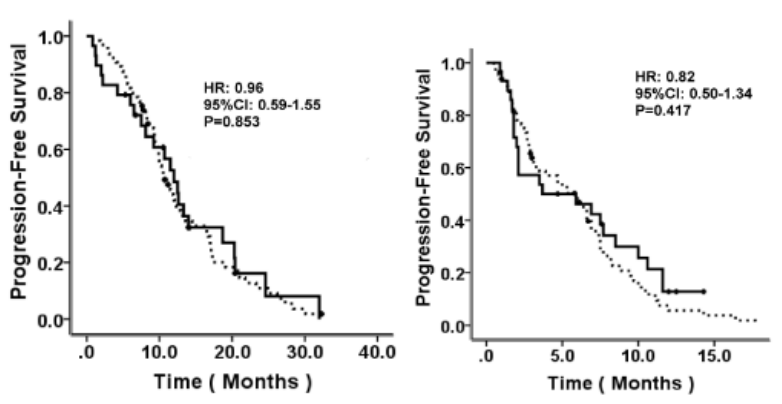

Figure 1. The Total PFS (PFSt) of the Whole Cohort (A), or CT (Solid Line) and TC (Dash Line) Group Respectively (B). Targeted therapy achieved a longer PFS over chemotherapy both in the initial therapy (C, solid line, chemotherapy, dash line, targeted therapy) and salvage therapy (D, solid line: targeted therapy, dash line, chemotherapy). PFS of targeted therapy was similar between the initial therapy (dash line) and salvage therapy (solid line, E). Also, PFS of chemotherapy was comparable in the initial (solid line) and salvage therapy (dash line, F). 
Table 1. Demographic Features of the Enrolled Patients

\begin{tabular}{|c|c|c|c|c|}
\hline N (\%) & $\begin{array}{c}\text { Total } \\
94(100)\end{array}$ & $\begin{array}{l}\text { TKI followed by chemotherapy } \\
\qquad 65(69.1)\end{array}$ & $\begin{array}{l}\text { Chemotherapy followed by TKI } \\
\qquad 23(30.9)\end{array}$ & $P$ value \\
\hline \multicolumn{5}{|l|}{ Sex } \\
\hline Female & $52(55.3)$ & $33(35.1)$ & $19(20.2)$ & \\
\hline Male & $42(44.7)$ & $32(34.1)$ & $10(10.6)$ & 0.71 \\
\hline \multicolumn{5}{|l|}{ Age } \\
\hline$\leq 60$ & $60(63.8)$ & $41(43.6)$ & $19(20.2)$ & \\
\hline$>60$ & $34(36.2)$ & $24(25.6)$ & $10(10.6)$ & 0.97 \\
\hline \multicolumn{5}{|l|}{ Smoking } \\
\hline Yes & 35 (37.2) & 25 (26.6) & $10(10.6)$ & \\
\hline No & $59(62.8)$ & $40(42.6)$ & $19(20.2)$ & 0.64 \\
\hline \multicolumn{5}{|l|}{ Performance } \\
\hline 0 & $48(51.1)$ & $34(36.2)$ & $14(14.9)$ & \\
\hline 1 & $46(48.9)$ & $31(33.0)$ & $15(15.9)$ & 0.12 \\
\hline \multicolumn{5}{|l|}{ Histology } \\
\hline $\mathrm{ADC}$ & 90 (95.7) & $62(66.0)$ & $28(29.7)$ & \\
\hline Non-ADC & $4(4.3)$ & $3(3.2)$ & $1(1.1)$ & 0.84 \\
\hline \multicolumn{5}{|l|}{ EGFR mutation } \\
\hline Exon 19 Del & $40(42.6)$ & $24(25.5)$ & $16(17.1)$ & \\
\hline L858R & $40(42.6)$ & $33(35.1)$ & $7(7.5)$ & \\
\hline Other & $14(14.8)$ & $8(8.5)$ & & 0.73 \\
\hline
\end{tabular}

Abbreviations, ADC; adenocarcinoma; Non-ADC, non-adenocarcinoma.

demographic features (Table 1).

No CR was observed. Chemotherapy achieved a similar disease control rate (DCR, CR and SD combined) in the initial and salvage therapy (69.0\% and $76.9 \%)$. Similarly, the DCR of TKI was comparable in its upfront and late use (93.8\% and $86.2 \%)$. The efficiency of TKI was consistently better than that of chemotherapy. Less PD was observed in both initial $(6.2 \%$ and $31.0 \%)$ and salvage therapy (13.8\% and $23.1 \%$, Figure 3$)$.

At the time of preparing this manuscript (Sept 2017), 81 patients $(86.2 \%)$ had progressed after both TKI and chemotherapy. The PFSt was similar between the CT group (16.1 mon, 95\% CI: 9.1-23.1 mon) and the TC group (18.0 mon, 95\% CI: 15.8-20.2 mon, HR 0.92, 95\% CI: $0.57-1.50, \mathrm{P}=0.75$, Figure $1 \mathrm{~B})$. A longer PFS was achieved for TKI over chemotherapy both in the initial (10.6 and 5.9 mon, HR 2.62, 95\% CI: 1.55-4.41 mon, $\mathrm{P}=0.001)$ and salvage therapy (12.0 and 6.0 mon, HR 1.29, 95\% CI: 0.78-2.14, $\mathrm{P}=0.001$, Figure $1 \mathrm{C}$ and D). TKI remained effective either before (10.6 mon) or after (12.0 mon) chemotherapy (HR 0.96, 95\% CI: $0.59-1.55, \mathrm{P}=0.853)$. The same trend was observed for

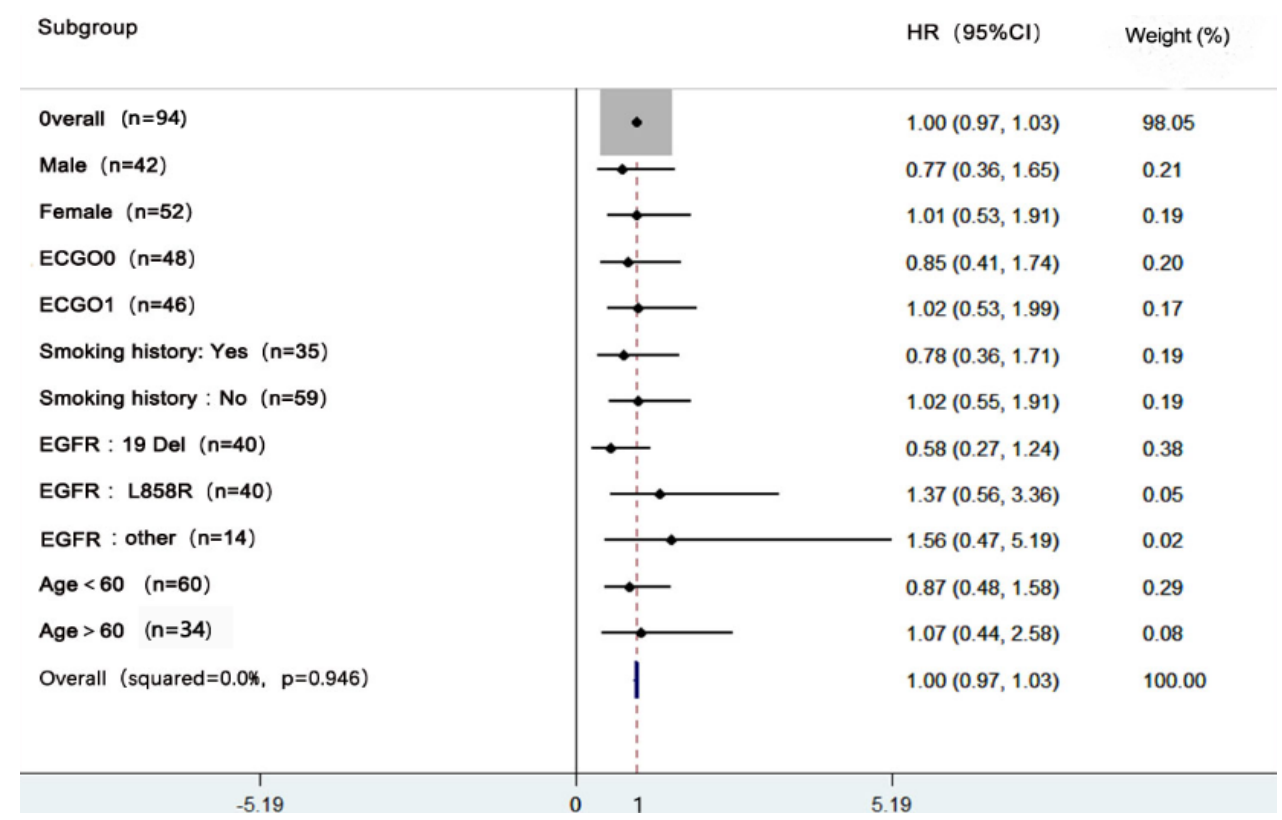

Figure 2. Subgroup Analysis of the PFSt 
A

B
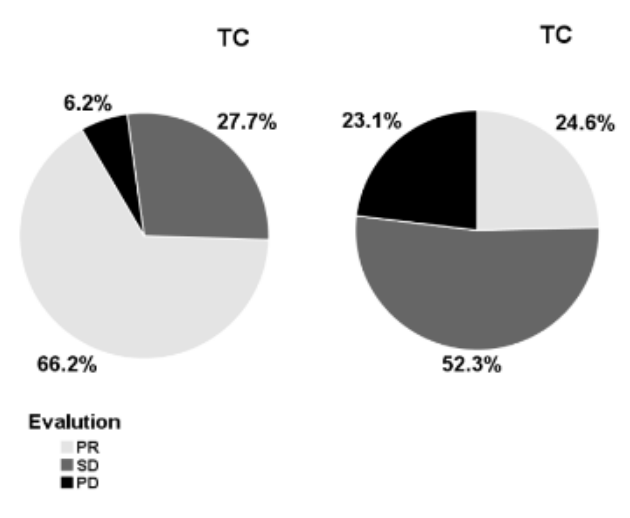

C

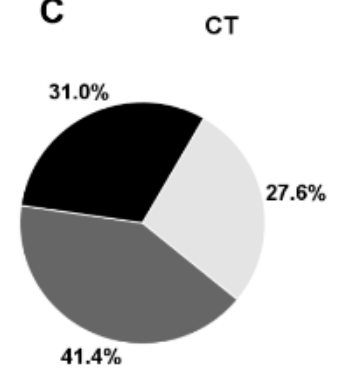

D

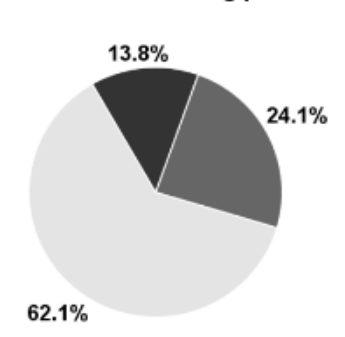

Figure 3. The Objective Responses of Chemotherapy or TKI in the TC (B and C) or CT Group (A and D) Therapy.

chemotherapy (5.9 and 6.0 mon for initial and salvage therapy, respectively, HR $0.82,95 \% \mathrm{CI}: 0.50-1.34$, $\mathrm{P}=0.417$, Figure $1 \mathrm{E}$ and $\mathrm{F}$ ). Most of our patients did not receive re-biopsy and the data on T790M resistance mutaion were lacking. Among 26 patients who had availabe T790M data, only 2 were found T790M(+) before chemotherapy. The low frequency of T790M $(+)$ wouldn't impact much on our conclusion.

\section{Discussion}

In this retrospective study, consecutive 1,682 patients with NSCLC were screened against the inclusion criteria and 94 patients were enrolled in this study. This study found the sequential administration of TKI and pemetrexed doublet chemotherapy to the patients with tumours harbouring EGFR mutations achieved a long PFS (18.0 mon), irrespective of the order of the sequence. It was also found the PFS for either TKI or chemotherapy were similar in both the initial or salvage therapy. TKI consistently achieved a longer PFS over chemotherapy in both lines of therapy.

The combination of TKI and chemotherapy was once considered inappropriate. The pilot ISEL study where gefitinib was combined with chemotherapy showed the combination increased toxicity with no beneficial effects (Thatcher et al., 2005). However, the study recruited unselected patients with no regard to EGFR mutation status. Later, the FASTACT-2 study showed that intercalated chemotherapy and erlotinib prolonged PFS over chemotherapy, and the benefit was mostly restricted to the cohort with EGFR mutations (Wu et al., 2013). The JMIT study suggested that the combination of pemetrexed-based chemotherapy and gefitinib

improved PFS compared with gefitinib monotherapy (15.8 and 10.9 mon) in patients with EGFR mutations. However, this study failed to provide information on the effects of pemetrexed and TKI administered sequentially. Any data about a direct comparison between concurrent and sequential use of TKI and pemetrexed chemotherapy were lacking. The total PFS of our study was similar to that of the JMIT study, which argues that the sequential administration of TKI and pemetrexed chemotherapy is also effective.

Given a comparable PFS, less toxicity would be expected in sequential compared with simultaneous administration of therapies. This hypothesis has been repeatedly confirmed in the FASTACT-2, JMIT, and other studies (Ahn et al., 2012). In addition, the failure of each therapy is easily recognizable and managed with ample reports in the sequential setting, which can be a challenge in simultaneous therapy. In certain situations, continuous TKI treatment beyond PD has been found to be beneficial (Tudor et al., 2017; Hsu et al., 2016). Lastly, the clinical schedule of each therapy was easily managed when they were used separately.

The long PFS achieved by the sequential administration has its underlying reasons. The PARAMOUNT study showed that first-line and maintenance pemetrexed doublet chemotherapy achieved a PFS of 7.9 mon (PazAres et al., 2013). TKI treatment has a durable PFS (10.8-13.1 mon) in first line therapy, as reported by numerous studies (Maemondo et al., 2010; Fukuoka et al., 2011; Zhou et al., 2011; Sequist et al., 2013). TKI and pemetrexed chemotherapy both have good control of tumour growth without possible interference from the other. Second, a potential synergy between pemetrexed chemotherapy and TKI might exist (Giovannetti et al., 2008). Low thymidylate synthase expression is predictive of pemetrexed efficacy, and gefitinib suppresses its expression in NSCLC cell lines (Chamizo et al., 2015; Kim et al., 2009). In addition, one preclinical report showed an erlotinib-induced G1-phase arrest protected these cells from the cytotoxicity of subsequent exposure to pemetrexed. Cell cycle redistribution also supports sequential administration in order to avoid the possible antagonism between these therapies.

Whether initial chemotherapy treatment influences the following response to TKI or vice versa has not been thoroughly elucidated to date. In our study, TKI as initial or salvage therapy achieved a similar PFS (10.6 and $12.0 \mathrm{mon})$, which was consistent with a previous report (Zeng et al., 2014). However, in that article, chemotherapy in the initial therapy had a longer PFS, opposite to this study's findings. The reasons behind this difference remain elusive. However, in the current study, only pemetrexed (but not other chemotherapy agents) was included. Pemetrexed might achieve a longer PFS among other chemotherapy agents, regardless of its use as frontline or behind-line treatment (Scagliotti et al., 2008; Yang et al., 2016; Sun et al., 2012). Additionally, synergistic effects between pemetrexed chemotherapy and TKI have been reported previously. The findings of these reports may help to explain the difference.

The current study has its limitations. This was 
a retrospective study conducted in a single institute. Selection bias is always inevitable in such studies and all of the conclusions require further confirmation. In addition, only PFS was included in this study, and data on survival were lacking because survival is often confounded by subsequent therapies. In addition, only patients with both TKI and pemetrexed chemotherapy were included in this study. Those without salvage therapy due to deleterious performance were excluded. This might lead to an overestimation of the treatment effects. These inclusion criteria were used because the aim of the study was to explore the effects of sequential administration of both therapies, and the cohort in this study was similar to that in the JMIT study.

In summary, the sequential administration of TKI and pemetrexed chemotherapy achieved a long duration of response in advanced NCLC patients with EGFR-mutant tumours, comparable to that of simultaneous administration reported in the JMIT study. The effects did not seem to be affected by the sequence of the therapy. The current study enrolled patients receiving both lines of therapies. Those not prescribed with any 2 nd line therapy due to death or poor performance were excluded. This might contribute to the observed longer PFS over that in JMIT study. Our study argues for the sequential administration of TKI and pemetrexed chemotherapy would be an option in this population.

\section{Acknowledgments}

This work was non-funded, and approved by Cancer Center, West China Hospital Sichuan University. All the authors declared no conflict of interest. The study was approved by the ethical committee of Sichuan University.

\section{References}

Ahn MJ, Yang JC, Liang J, et al (2012). Randomized phase II trial of first-line treatment with pemetrexed-cisplatin, followed sequentially by gefitinib or pemetrexed, in East Asian, never-smoker patients with advanced non-small cell lung cancer. Lung Cancer, 77, 346-52.

Chamizo C, Zazo S, Domine M, et al (2015). Thymidylate synthase expression as a predictive biomarker of pemetrexed sensitivity in advanced non-small cell lung cancer. $B M C$ Pulm Med, 15, 132.

Cheng Y, Murakami H, Yang PC, et al (2016). Randomized phase II trial of Gefitinib with and without pemetrexed as first-line therapy in patients with advanced non-squamous non-small-cell lung cancer with activating epidermal growth factor receptor mutations. J Clin Oncol, 34, 3258-66.

Eisenhauer EA, Therasse P, Bogaerts J, et al (2009). New response evaluation criteria in solid tumours: revised RECIST guideline (version 1.1). Eur J Cancer, 45, 228-47.

Fukuoka M, Wu YL, Thongprasert S, et al (2011). Biomarker analyses and final overall survival results from a phase III, randomized, open-label, first-line study of gefitinib versus carboplatin/paclitaxel in clinically selected patients with advanced non-small-cell lung cancer in Asia (IPASS). J Clin Oncol, 29, 2866-74.

Giovannetti E, Lemos C, Tekle C, et al (2008). Molecular mechanisms underlying the synergistic interaction of erlotinib, an epidermal growth factor receptor tyrosine kinase inhibitor, with the multitargeted antifolate pemetrexed in non-small-cell lung cancer cells. Mol Pharmacol, 73, 1290-300.

Hirsch FR, Scagliotti GV, Mulshine JL, et al (2009). Lung cancer: current therapies and new targeted treatments. Lancet, $\mathbf{3 8 9}$, 299-311.

Hsu PC, Liu CY, Li SH, et al (2016). Efficacy of platinumbased combination chemotherapy in advanced lung adenocarcinoma harboring sensitive epidermal growth factor receptor (EGFR) mutations with acquired resistance to first-line EGFR tyrosine kinase inhibitor (TKI). Cancer Treat Res Commun, 9, 48-55.

Kim HP, Yoon YK, Kim JW, et al (2009). Lapatinib, a dual EGFR and HER2 tyrosine kinase inhibitor, downregulates thymidylate synthase by inhibiting the nuclear translocation of EGFR and HER2. PLoS One, 4, e5933.

Lee DH, Lee JS, Kim SW, et al (2013). Three-arm randomised controlled phase 2 study comparing pemetrexed and erlotinib to either pemetrexed or erlotinib alone as second-line treatment for never-smokers with non-squamous non-small cell lung cancer. Eur J Cancer, 49, 3111-21.

Maemondo M, Inoue A, Kobayashi K, et al (2010). Gefitinib or chemotherapy for non-small-cell lung cancer with mutated EGFR. N Engl J Med, 362, 2380-8.

Mok TS, Wu YL, Yu CJ, et al (2009). Randomized, placebo-controlled, phase II study of sequential erlotinib and chemotherapy as first-line treatment for advanced non-small-cell lung cancer. J Clin Oncol, 27, 5080-7.

Paez JG, Janne PA, Lee JC, et al (2004). EGFR mutations in lung cancer: Correlation with clinical response to gefitinib therapy. Science, 304, 1497-1500.

Paz-Ares LG, de Marinis F, Dediu M, et al (2013). PARAMOUNT: Final overall survival results of the phase III study of maintenance pemetrexed versus placebo immediately after induction treatment with pemetrexed plus cisplatin for advanced non-squamous non-small-cell lung cancer. J Clin Oncol, 31, 2895-902.

Rice TW, Blackstone EH, Rusch VW, et al (2010). 7th edition of the AJCC Cancer Staging Manual: esophagus and esophagogastric junction. Ann Surg Oncol, 17, 1721-4.

Scagliotti GV, Parikh P, von Pawel, J et al (2008). Phase III study comparing cisplatin plus gemcitabine with cisplatin plus pemetrexed in chemotherapy-naive patients with advanced-stage non-small-cell lung cancer. J Clin Oncol, 26, 3543-51.

Sequist LV, Yang JC, Yamamoto N, et al (2013). Phase III study of afatinib or cisplatin plus pemetrexed in patients with metastatic lung adenocarcinoma with EGFR mutations. J Clin Oncol, 31, 3327-34.

Siegel RL, Miller KD, Jemal A, et al (2018). Cancer statistics. CA Cancer J Clin, 68, 7-30.

Sun JM, Lee KH, Kim SW, et al (2012). Gefitinib versus pemetrexed as second-line treatment in patients with non-small cell lung cancer previously treated with platinum-based chemotherapy (KCSG-LU08-01): an open-label, phase 3 trial. Cancer, 118, 6234-42.

Thatcher N, Chang A, Parikh P, et al (2005). Gefitinib plus best supportive care in previously treated patients with refractory advanced non-small-cell lung cancer: results from a randomised, placebo-controlled, multicentre study (Iressa Survival Evaluation in Lung Cancer). Lancet, 366, 1527-37.

Torre LA, Siegel RL, Ward EM, et al (2016). Global cancer incidence and mortality rates and trends-An update. Cancer Epidemiol Biomarkers Prev, 25, 16-27.

Tudor RA, D'Silva A, Tremblay A, et al (2017). Beyond disease-progression: Clinical outcomes after EGFR-TKIs in a cohort of EGFR mutated NSCLC patients. PLoS One, 


$$
\text { 12, e0181867. }
$$

Wu YL, Lee JS, Thongprasert S, et al (2013). Intercalated combination of chemotherapy and erlotinib for patients with advanced stage non-small-cell lung cancer (FASTACT-2): a randomised, double-blind trial. Lancet Oncol, 14, 777-86.

Wu YL, Lu S, Cheng Y, et al (2014). Efficacy and safety of pemetrexed/cisplatin versus gemcitabine/cisplatin as first-line treatment in Chinese patients with advanced non-squamous non-small cell lung cancer. Lung Cancer, 85, 401-7.

Yang CJ, Tsai MJ, Hung JY, et al (2016). Pemetrexed had significantly better clinical efficacy in patients with stage IV lung adenocarcinoma with susceptible EGFR mutations receiving platinum-based chemotherapy after developing resistance to the first-line gefitinib treatment. Onco Targets Ther, 9, 1579-87.

Yang JC, Srimuninnimit V, Ahn MJ, et al (2016). First-line pemetrexed plus Cisplatin followed by Gefitinib maintenance therapy versus Gefitinib monotherapy in East Asian never-smoker patients with locally advanced or metastatic nonsquamous non-small cell lung cancer: Final overall survival results from a randomized phase 3 study. J Thorac Oncol, 11, 370-9.

Zeng Z, Yan HH, Zhang XC, et al (2014). Reduced chemotherapy sensitivity in EGFR-mutant lung cancer patient with frontline EGFR tyrosine kinase inhibitor. Lung Cancer, 86, 219-24.

Zhou C, Wu YL, Chen G, et al (2015). Final overall survival results from a randomised, phase III study of erlotinib versus chemotherapy as first-line treatment of EGFR mutationpositive advanced non-small-cell lung cancer (OPTIMAL, CTONG-0802). Ann Oncol, 26, 1877-83.

Zhou C, Wu Y-L, Chen G, Feng J, et al (2011). Erlotinib versus chemotherapy as first-line treatment for patients with advanced EGFR mutation-positive non-small-cell lung cancer (OPTIMAL, CTONG-0802): a multicentre, openlabel, randomised, phase 3 study. Lancet Oncol, 12, 735-42.

This work is licensed under a Creative Commons AttributionNon Commercial 4.0 International License. 